\title{
Frequency modulation response due to the intensity modulation of fiber-grating Fabry- Perot lasers
}

\begin{abstract}
A comprehensive study on the small-signal frequency modulation (FM) characteristics of a fiber-grating Fabry-Perot (FGFP) laser is numerically conducted. Fiber Bragg grating (FBG) is used as a wavelength selective element to control the properties of the laser output by controlling the external optical feedback (OFB) level. In addition to the external OFB level, the effect of other parameters such as temperature, injection current, cavity volume, nonlinear gain compression factor, linewidth enhancement factor, and fiber-grating (FG) parameters on FM characteristics of the laser are investigated. The study is performed by modifying a set of rate equations that are solved by considering the effects of external OFB and ambient temperature (T) variations. The temperature dependence (TD) of FM characteristics is calculated according to TD of laser parameters instead of using well-known Pankove relationship. Results show that the optimum external fiber length (Lext) is $3.1 \mathrm{~cm}$ and the optimum range of working temperature for FGFP laser is within $\pm 2{ }^{\circ} \mathrm{C}$ from the $\mathrm{FBG}$ reference temperature (To). Also, it is shown that antireflection (AR) coating reflectivity and the linewidth enhancement factor have no significant effect on the FM response. The FM spectra peak amplitude is less than $5 \mathrm{~dB}$ with $5 \mathrm{~mW}$ output power. Good temperature stability is also obtained.
\end{abstract}

Keyword: External cavity semiconductor lasers; External optical feedback; Fiber Bragg grating; Frequency modulation 